\title{
Fair-Sized Projective Modules
}

\author{
PAVEL PŘíHoda (*)
}

\begin{abstract}
We investigate a condition on particular chains of ideals that allows us to determine properties of infinitely generated modules over noetherian rings. The results apply to semilocal noetherian rings, integral group rings of finite groups and universal enveloping algebras of solvable Lie algebras of finite dimension.
\end{abstract}

\section{Introduction.}

This paper is devoted to the study of infinitely generated projective modules over associative unitary rings. We are interested in the case in which the ring has projective modules that are not direct sums of finitely generated modules. Some general results and examples of rings with such modules were given in [12]. Our motivation was to find a technique that could be applied to prove the existence of superdecomposable projective modules over semilocal rings.

Let us briefly explain the main idea of the paper. According to a well known theorem of Kaplansky, any projective right module over a ring $R$ is a direct sum of countably generated right modules, so it suffices to investigate countably generated projectives, that is, direct summands of a countably generated free right module $F=R_{R}^{(\mathrm{N})}$. Suppose that $P \oplus P^{\prime}=F$. The canonical projection $\pi: F \rightarrow P$ is given by a column-finite $\mathbb{N} \times \mathbb{N}$ idempotent matrix $A$. We say that $A$ represents $P$ (observe that the columns of $A$ generate $P$ ). Let $I_{n}$ be the ideal of $R$ generated by the entries of $A$ that are below the $n$-th row. Clearly, $P$ is finitely generated if and only if there exists $k \in \mathbb{N}$ such that $I_{l}=0$ for every $l \geq k$. The other possible extreme case is when $I_{1}=I_{2}=\cdots=R$. It is a well-known result of Bass

(*) Indirizzo dell'A.: Charles University in Prague, Faculty of Mathematics and Physics, Department of Algebra, Sokolovská 83, 18675 Prague, Czech Republic.

E-mail: paya@matfyz.cz

Supported by Eduard Čech center for algebra and geometry, grant LC505. 
[3, Theorem 3.1] that in this case $P \simeq F$ provided $R / J(R)$ is right noetherian. In this paper, we focus our attention on the case in which the sequence $I_{1} \supseteq I_{2} \supseteq \ldots$ terminates at an ideal $I$. It is easy to see that $I$ is idempotent. We show that if $R$ is left and right noetherian and the sequence $I_{1} \supseteq I_{2} \supseteq \cdots$ terminates at $I$, then $P$ contains as a direct summand any countably generated projective module having its trace ideal in $I$. Cf. [3, Theorem 3.1]. The following easy condition assures that any sequence of ideals derived from an idempotent column-finite matrix terminates: If $I_{1}, I_{2}, \ldots$ is a sequence of ideals in $R$ such that $I_{k+1} I_{k}=I_{k+1}$ for any $k \geq 1$, then there exists $n$ such that $I_{n}=I_{n+1}=\ldots$ Call (*) this condition.

In section 2 , we show that over a left and right noetherian ring $R$ satisfying condition (*), the theory of projective modules "reduces" to the theory of idempotent ideals in $R$ and the theory of finitely generated projective modules over the factor rings of $R$ modulo idempotent ideals. This explains and is related to the statement in the introduction of [3], according to which "infinitely generated projective modules invite little interest".

The remaining sections are devoted to presenting some examples. We prove that $(*)$ holds for semilocal noetherian rings, integral group rings of a finite group and universal enveloping algebras of finite solvable Lie algebras over a field of characteristic zero. This allows us to prove that:

(i) There exists a semilocal noetherian ring with superdecomposable projective modules.

(ii) Indecomposable projective modules over integral group rings of finite groups are finitely generated.

(iii) Any infinitely generated projective module over a finite dimensional solvable Lie algebra over a field of characteristic zero is free.

Notice that (ii) solves [9, Problem 8.34].

Let us briefly recall some notions and fix the notation. The word "ring" always means associative ring with an identity and "module" means unital right module. If $M$ is a module over $R$, then $\sum_{f \in \operatorname{Hom}_{R}(M, R)} f(M)$ is an ideal of $R$ called the trace ideal of $M$. We denote it $\operatorname{Tr}(M)$. If $P$ is a projective module over $R$, then $\operatorname{Tr}(P)$ is the smallest ideal $X$ of $R$ such that $P X=P$, and is an idempotent ideal. Further if $X$ is a subset of a ring $R$, we denote $R X R$ the (two-sided) ideal generated by $X$. In case $X=\{x\}$ we denote $R x R$ the smallest ideal of $R$ containing $x$. Notice that in general the relation $R x R=\{r x s \mid r, s \in R\}$ does not hold. Recall the following important result due to Whitehead: 
FACT 1.1 [18, Corollary 2.7]. Let I be an idempotent ideal of $R$ finitely generated on the left. Then there exists a countably generated projective right $R$-module $P$ such that $\operatorname{Tr}(P)=I$.

To avoid confusion, we will call the rings which have all left ideals and all right ideals finitely generated left and right noetherian rings, although they are often called noetherian rings. Finally, we will call infinitely generated projective modules the projective modules that are countably generated but not finitely generated.

\section{2. $I$-big modules}

Let $P$ be a countably generated projective module over a ring $R$ and let $I$ be an ideal of $R$. We say that $P$ is $I$-big if for any countably generated projective module $Q$ with trace ideal contained in $I$ there exists an epimorphism of $P$ onto $Q$. Hence, in this case, $P$ contains a direct summand isomorphic to $Q$. Notice that this definition will be applied to countably generated projective modules only.

REMARK 2.1 (Eilenberg's trick). Let $I$ be an ideal of a ring $R$ and let $P$ be an $I$-big projective module. If $Q$ is a countably generated projective module with trace ideal contained in $I$, then $P \oplus Q \simeq P$, because $Q^{(\omega)}$ is a direct summand of $P$.

LEMMA 2.2. Let I be an idempotent ideal that is finitely generated as a left ideal. Then there exists an I-big projective module $P$ such that $\operatorname{Tr}(P)=I$. Such a module $P$ is unique up to isomorphism.

Proof. By [18, Corollary 2.7], there exists a countably generated projective module $P$ with $\operatorname{Tr}(P)=I$. Clearly, $\operatorname{Tr}\left(P^{(\omega)}\right)=I$. If $Q$ is a countably generated projective module having the trace ideal contained in $I$, then $Q I=Q$ and $Q$ is a factor of $P^{(\omega)}$. Let $P_{1}, P_{2}$ be $I$-big modules such that $\operatorname{Tr}\left(P_{1}\right)=\operatorname{Tr}\left(P_{2}\right)=I$. By Remark 2.1, $P_{1} \oplus P_{2} \simeq P_{1}$. Similarly, $P_{1} \oplus P_{2} \simeq P_{2}$. Thus $P_{1} \simeq P_{2}$.

REMARK 2.3. We have just proved that, for any ideal $I$ that is a trace ideal of a countably generated projective module, there exists a unique countably generated projective module $P$ (up to isomorphism) such that $P$ is $I$-big and $\operatorname{Tr}(P)=I$. We will make use of $I$-big modules over left and 
right noetherian rings. Observe that $R^{(\omega)}$ is an $R$-big projective module and that any $R$-big projective module has trace ideal $R$. Therefore any $R$-big projective module is isomorphic to $R^{(\omega)}$.

We say that a ring $R$ satisfies Condition (*) if for any sequence $I_{1}, I_{2}, \ldots$ of ideals in $R$ such that $I_{k+1} I_{k}=I_{k+1}, k \in \mathbb{N}$ there exists $n \in \mathbb{N}$ such that $I_{k}=I_{n}$ for any $n \leq k \in \mathbb{N}$. Notice that such a sequence is necessarily a descending chain.

We will use this condition in the following context: Suppose we have a countably generated projective module $P$. Thus $P$ is a direct summand of a countably generated free module, $P \oplus P^{\prime}=R^{(\mathrm{N})}$ say. The canonical projection $\pi: R^{(\mathrm{N})} \rightarrow P$ can be written with respect to the canonical basis of $R^{(\mathrm{N})}$ as an $\mathbb{N} \times \mathbb{N}$ matrix $A=\left(a_{i, j}\right)_{i, j \in \mathbb{N}}$ with entries in $R$. Moreover, $A$ is a column-finite matrix (that is, for any $j \in \mathbb{N}$ there exists $i \in \mathbb{N}$ with $a_{k, j}=0$ whenever $k \geq i$ ). Therefore $A^{2}$ is defined and it is easy to see that $A^{2}=A$ (that is, $A$ is an idempotent matrix). Conversely, given any idempotent column-finite $\mathbb{N} \times \mathbb{N}$ matrix $A$, the corresponding module $P=A R^{(\mathrm{N})}$ is projective.

Now, let $A=\left(a_{i, j}\right)_{i, j \in \mathrm{N}}$ be an idempotent column-finite matrix over $R$ and let $I_{k}=\sum_{k \leq i \in \mathbb{N}, j \in \mathbb{N}} R a_{i, j} R, k \in \mathbb{N}$. For any $k \in \mathbb{N}$ there exists an integer $n_{k}>k$ such that $a_{i, j}=0$ whenever $i \geq n_{k}$ and $j<k$. Since $A$ is idempotent, we have $I_{n_{k}} I_{k}=I_{n_{k}}$. Hence there exist positive integers $m_{1}<m_{2}<\cdots$ such that $I_{m_{j+1}} I_{m_{j}}=I_{m_{j+1}}$. If $R$ satisfies (*), then there exists $l \in \mathbb{N}$ such that $I_{m_{j}}=I_{m_{l}}$ for any $l \leq j \in \mathbb{N}$, in particular, $I_{m_{j}}=I_{m_{j+1}}=I_{m_{j+1}} I_{m_{j}}=I_{m_{j}}^{2}$ if $j \geq l$. So if $I=\cap_{j \in \mathbb{N}} I_{n_{j}}$, then $I$ is an idempotent ideal and $I_{j}=I$ for almost all $j \in \mathbb{N}$.

We will say that a projective module $P$ over a ring $R$ is fair-sized if $P$ is countably generated and the set $I(P):=\{I \mid I$ is an ideal of $R$ such that $P / P I$ is finitely generated $\}$ has a least element. The following lemma shows that any countably generated projective module over a ring satisfying (*) is fair-sized. Moreover, the proof reveals the relation between the smallest element of $I(P)$ and an idempotent matrix representing $P$.

LEMMa 2.4. Let $R$ be a ring satisfying (*) and let $P$ be a countably generated projective module over $R$. The set $\{I \mid I$ is an ideal of $R$ such that $P / P I$ is finitely generated $\}$ has a least element $I_{0}$, which is an idempotent ideal.

Proof. Let $A=\left(a_{i, j}\right)_{i, j \in \mathrm{N}}$ be an idempotent column-finite matrix representing $P$, and $I_{k}, k \in \mathbb{N}$, be the ideals defined above. Set 
$I_{0}=\cap_{k \in \mathbb{N}} I_{k}$. As remarked above, $I_{0}$ is idempotent. Let $\left\{e_{i} \mid i \in \mathbb{N}\right\}$ be the canonical free basis of $R^{(\mathrm{N})}$ and suppose that $I_{0}=I_{m}=I_{m+1}=\ldots$ Then $\sum_{i=1}^{m-1} A e_{i} R+P I_{0}=P$, so $P / P I_{0}$ is finitely generated. Let $K$ be an ideal such that $P / P K$ is a finitely generated module. Assume $P=A R^{(\mathrm{N})} \subseteq R^{(\mathrm{N})}$. Notice that $P K=P \cap K^{(\mathrm{N})}$, that is, the elements of $P K$ are exactly the elements of $P$ having all their components in $K$. If $P / P K$ is finitely generated, then there exists $k \in \mathbb{N}$ such that $a_{i, j} \in K$ for every $i \geq k$ and $j \in \mathbb{N}$. Therefore $I_{0} \subseteq K$.

Thus if $R$ satisfies (*), every countably generated projective module $P$ determines a pair $\left(I, P^{\prime}\right)$, where $I$ is an idempotent ideal and $P^{\prime}$ is a finitely generated projective module over $R / I$. More precisely, $I$ is the smallest ideal of $R$ such that $P / P I$ is finitely generated and $P^{\prime}$ is the module $P / P I$ considered as an $R / I$-module in the obvious way. If $P$ is a countably generated projective module, then the corresponding idempotent ideal $I$ is given by a matrix representing $P$ as $I=\cap_{k \in \mathbb{N}} I_{k}$, but the characterization of $I$ in Lemma 2.4 implies that $I$ is independent of the choice of the matrix (and of the complement $P^{\prime}$ in the decomposition $P \oplus P^{\prime}=R^{(\mathrm{N})}$ ).

Lemma 2.5. Let I be an idempotent ideal of a ring $R$ such that I is finitely generated as a left module and as a right module. If $P$ and $Q$ are I-big projective modules satisfying $P / P I \simeq Q / Q I$, then $P \simeq Q$.

Proof. Let $B$ be the unique $I$-big projective module having trace ideal $I$. Observe that $P \oplus B^{(\omega)} \simeq P$ by Remark 2.1. If $f: P \rightarrow Q$ induces an isomorphism $P / P I \rightarrow Q / Q I$, then $f(P)+Q I=Q$. Since $Q I$ is countably generated and $\operatorname{Tr}(B)=I$, we get an epimorphism $h: P \oplus B^{(\omega)} \rightarrow Q$ such that $\left.h\right|_{P}=f$. As $f$ induces a monomorphism $P / P I \rightarrow Q / Q I$ and $h\left(B^{(\omega)}\right) \subseteq Q I$, we get $X=\operatorname{Ker} h \subseteq P I \oplus B^{(\omega)}$. Thus $X$ is a direct summand of $P I \oplus B^{(\omega)}$. In particular, $X I=X$. Consequently, $\operatorname{Tr}(X) \subseteq I$, so $Q \oplus X \simeq Q$ by Remark 2.1. Finally, $Q \simeq Q \oplus X \simeq P \oplus B^{(\omega)} \simeq P$, and $Q \simeq P$ follows.

The following lemma is a straightforward extension of [18, Corollary 2.7].

LEMMa 2.6. Let I be a proper idempotent ideal of a ring R. Assume I finitely generated as a left ideal. Let $P^{\prime}$ be a finitely generated projective module over $R / I$. Then there exists an I-big projective module $P$ such that $P / P I \simeq P^{\prime}$. 
Proof. We will find a countably generated projective module $P_{0}$ such that $P_{0} / P_{0} I \simeq P^{\prime}$.

Suppose that $P^{\prime}$ is given by an $n \times n$ matrix $X$ idempotent modulo $I$. The $R$-matrix $X$ is a lifting of an idempotent $R / I$-matrix $\bar{X}$. Let $I=I i_{1}+\cdots+I i_{l}, i_{1}, \ldots, i_{l} \in I$. Construct a sequence of matrices $A_{1}, A_{2}, \ldots$ as follows: $A_{1}$ has $c_{1}=n$ columns and $r_{1}=l n+n$ rows. The square matrix given by the first $n$ rows of $A_{1}$ is $X,\left(A_{1}\right)_{i, j}=0$ if $n<i \leq n+(j-1) l$ or $i>n+j l$, and the remaining entries in each column are filled with the generators $i_{1}, \ldots, i_{l}$. That is, the matrix $A_{1}$ written in blocks is

$$
\left(\begin{array}{ccccc} 
& & X & & \\
b & 0 & 0 & \cdots & 0 \\
0 & b & 0 & \cdots & 0 \\
& & & \vdots & \\
0 & 0 & 0 & \cdots & b
\end{array}\right),
$$

where $b$ is the column $\left(i_{1}, \ldots, i_{l}\right)^{T}$.

If $A_{k}, r_{k}, c_{k}$ have been defined, then $A_{k+1}$ has $c_{k+1}=r_{k}$ columns and $r_{k+1}=r_{k}+l r_{k}$ rows. The $n \times n$ top left corner of $A_{k+1}$ is given by the matrix $X$ and all the other entries in the first $r_{k}$ rows of $A_{k+1}$ are zero. The remaining $l r_{k}$ rows contains $i_{1}, \ldots, i_{l}$ placed in each column in the same "independent manner" as described for $A_{1}$.

We claim that for any $k \in \mathbb{N}$ there is a $c_{k+1} \times r_{k+1}$ matrix $B_{k}$ such that $B_{k} A_{k+1} A_{k}=A_{k}$. Observe that the $c_{k} \times c_{k}$ matrix given by the first $c_{k}$ rows of $A_{k}$ is idempotent modulo $I$. We can find an $r_{k} \times r_{k}$ matrix $C_{k}$ such that $C_{k} A_{k}=A_{k}$ : The $n \times n$ top left corner of $C_{k}$ is given by $X$, the other entries in the first $c_{k}$ columns are zero and the matrix $C_{k}$ can be completed by elements of $I$ because $I=I i_{1}+\cdots+I i_{l}$ and $i_{1}, \ldots, i_{l}$ are placed independently in the bottom part of $A_{k}$. This matrix $C_{k}$ can be written as $D_{k} A_{k+1}$, where $D_{k}$ is a suitable $r_{k} \times r_{k+1}$ matrix. (Again we place $X$ in the top left corner of $D_{k}$, and put all the other entries in the first $r_{k}$ columns of $D_{k}$ equal to zero. The remaining entries can be completed because the generators of $I$ are placed independently in $A_{k+1}$.) Now, since $A_{k}=C_{k} A_{k}=D_{k} A_{k+1} A_{k}$, put $B_{k}=D_{k}$.

View the free module $F_{k}=R^{c_{k}}$ as the set of columns of length $c_{k}$. Let $f_{k}: F_{k} \rightarrow F_{k+1}$ be the homomorphism given by $f_{k}(u)=A_{k} \cdot u$ for every $u \in F_{k}$. By [18, Theorem 2.1], the colimit of the direct system induced by the $f_{k}$ 's is a projective module $P_{0}$. Obviously, $P_{0}$ is a countably generated module. Applying the functor $-\otimes_{R} R / I: \operatorname{Mod}-R \rightarrow \operatorname{Mod}-R / I$, we see that 
$P_{0} / P_{0} I$ is an $R / I$-module isomorphic to the colimit of the system $(R / I)^{n} \stackrel{\bar{X}}{\rightarrow}(R / I)^{n} \stackrel{\bar{X}}{\rightarrow} \cdots$, which is easily seen to be $\bar{X}(R / I)^{n} \simeq P^{\prime}$. Therefore $P_{0} / P_{0} I \simeq P^{\prime}$.

Finally, by Lemma 2.2, there exists an $I$-big projective module $B$ such that $\operatorname{Tr}(B)=I$. Since $B I=I, P:=P_{0} \oplus B$ is an $I$-big projective module with $P / P I \simeq P_{0} / P_{0} I \simeq P^{\prime}$.

REMARK 2.7. Let us explain the construction in the proof of Lemma 2.6 via an example. Suppose that $I$ is a proper idempotent ideal of a ring $R$ such that $I=I i_{1}+I i_{2}$ for some $i_{1}, i_{2} \in I$. Let $x \in R$ be such that $x+I$ is an idempotent element of $R / I$, i.e., $x-x^{2} \in I$. Then there are $t_{1}, t_{2} \in I$ such that $x=x^{2}+t_{1} i_{1}+t_{2} i_{2}$. Further, there are $u_{1}, u_{2}, v_{1}, v_{2} \in I$ such that $u_{1} i_{1}+u_{2} i_{2}=i_{1}$ and $v_{1} i_{1}+v_{2} i_{2}=i_{2}$. Set

$$
A_{1}=\left(\begin{array}{c}
x \\
i_{1} \\
i_{2}
\end{array}\right) \quad C_{1}=\left(\begin{array}{ccc}
x & t_{1} & t_{2} \\
0 & u_{1} & u_{2} \\
0 & v_{1} & v_{2}
\end{array}\right) \quad C_{1}^{\prime}=\left(\begin{array}{ccc}
x-x^{2} & t_{1} & t_{2} \\
0 & u_{1} & u_{2} \\
0 & v_{1} & v_{2}
\end{array}\right) .
$$

Obviously, $C_{1} A_{1}=A_{1}$. Moreover, all entries of $C_{1}^{\prime}$ are in $I$. Therefore there is a $3 \times 6$ matrix $T=\left(t_{i, j}\right)_{1 \leq i \leq 3,1 \leq j \leq 6}$ satisfying $T A_{2}^{\prime}=C_{1}^{\prime}$, where

$$
A_{2}^{\prime}=\left(\begin{array}{cccccc}
i_{1} & i_{2} & 0 & 0 & 0 & 0 \\
0 & 0 & i_{1} & i_{2} & 0 & 0 \\
0 & 0 & 0 & 0 & i_{1} & i_{2}
\end{array}\right)^{T}
$$

All the entries of $T$ can be chosen in $I$, but this is not important. It is easy to see that $C_{1}=B_{1} A_{2}$, where

$$
\begin{gathered}
B_{1}=\left(\begin{array}{ccccccccc}
x & 0 & 0 & t_{1,1} & t_{1,2} & t_{1,3} & t_{1,4} & t_{1,5} & t_{1,6} \\
0 & 0 & 0 & t_{2,1} & t_{2,2} & t_{2,3} & t_{2,4} & t_{2,5} & t_{2,6} \\
0 & 0 & 0 & t_{3,1} & t_{3,2} & t_{3,3} & t_{3,4} & t_{3,5} & t_{3,6}
\end{array}\right) \\
A_{2}=\left(\begin{array}{ccccccccc}
x & 0 & 0 & i_{1} & i_{2} & 0 & 0 & 0 & 0 \\
0 & 0 & 0 & 0 & 0 & i_{1} & i_{2} & 0 & 0 \\
0 & 0 & 0 & 0 & 0 & 0 & 0 & i_{1} & i_{2}
\end{array}\right)^{T} .
\end{gathered}
$$

The following lemma is, in a sense, a restatement of [3, Theorem 3.1]. We prefer to give a brief but complete proof of the statement for left and right noetherian rings rather than specifying what should be modified in the proof of [3, Theorem 3.1] to get a real generalization. 
LEMMA 2.8. Let $R$ be a left and right noetherian ring. Let $A=\left(a_{i, j}\right)_{i, j \in \mathrm{N}}$ be an idempotent column-finite matrix. Set $I_{k}=\sum_{i \geq k, j \in \mathbb{N}} R a_{i, j} R$. If there exists $n_{0} \in \mathbb{N}$ such that $I_{m}=I_{n_{0}}$ for every $m \geq n_{0}$, then the module $P=A R^{(\mathrm{N})} \subseteq R^{(\mathrm{N})}$ is $I_{n_{0}}$-big.

Proof. Set $I=I_{n_{0}}$ and observe that $I$ is finitely generated as a left ideal. Let $a_{i}$ be the $i$-th column of $A$. We will prove the following claim. For any $n \in \mathbb{N}$ there exist $m \in \mathbb{N}$ and $r_{1}, \ldots, r_{m} \in R$ such that if $a_{1} r_{1}+\cdots+a_{m} r_{m}=\left(c_{i}\right)_{i \in \mathbb{N}}$, then $I \subseteq \sum_{i \geq n} R c_{i}$. By induction, define positive integers $s_{1}, \ldots, s_{k}, s_{1}^{\prime}, \ldots, s_{k}^{\prime}$ and $x_{1}, \ldots, x_{k} \in R$ such that $\sum_{i=1}^{l} R x_{i} \subsetneq \sum_{i=1}^{l+1} R x_{i}$ for every $1 \leq l<k$ and $I \subseteq \sum_{i=1}^{k} R x_{i}$.

Put $s_{1}=1, s_{1}^{\prime}=n$ and $x_{1}=a_{s_{1}^{\prime}, s_{1}}$. If $I \subseteq R x_{1}$, we have finished. Otherwise, suppose we have defined positive integers $s_{1}, \ldots, s_{l}, s_{1}^{\prime}, \ldots, s_{l}^{\prime}$ and $x_{1}, \ldots, x_{l} \in R$ such that $I \nsubseteq \sum_{i=1}^{l} R x_{i}$. Since $R$ is right noetherian, there exists $m_{l} \in \mathbb{N}$ such that $m_{l}>s_{l}$ and $\sum_{j \in \mathbb{N}} a_{s_{l}^{\prime}, j} R=\sum_{1 \leq j<m_{l}} a_{s_{l}^{\prime}, j} R$. Since $A$ is column-finite, there exists $m_{l}^{\prime} \in \mathbb{N}$ with $m_{l}^{\prime}>s_{l}^{\prime}$ and $a_{i, j}=0$ whenever $i \geq m_{l}^{\prime}$ and $j \leq m_{l}$. As $I \subseteq I_{m_{l}^{\prime}}$, there exist $s_{l+1}^{\prime}>m_{l}^{\prime}, s_{l+1}>m_{l}$ and $t_{l+1} \in R$ such that $a_{s_{l+1}^{\prime}, s_{l+1}} t_{l+1} \notin \sum_{i=1}^{l} R x_{i}$. Put $x_{l+1}=a_{s_{l+1}^{\prime}, s_{l+1}} t_{l+1}$.

Since $R$ is left noetherian, this process must stop, that is, there exists $k$ such that $I \subseteq \sum_{1 \leq i \leq k} R x_{i}$. It follows that there are $r_{1}, \ldots, r_{s_{k}} \in R$ such that the $s_{i}^{\prime}$-th component of $\sum_{i=1}^{s_{k}} a_{i} r_{i}$ is $x_{i}$ for any $1 \leq i \leq k$. This is obvious for $k=1$. If $k>1$, note that $s_{1}<m_{1}<s_{2}<m_{2}<\cdots<m_{k-1}<s_{k}$ and $s_{1}^{\prime}<m_{1}^{\prime}<s_{2}^{\prime}<m_{2}^{\prime}<\cdots<m_{k-1}^{\prime}<s_{k}^{\prime}$. Further, $\sum_{j \in \mathbb{N}} a_{s_{1}^{\prime}, j} R=\sum_{j=1}^{m_{1}-1} a_{s_{1}^{\prime}, j} R$ and $\sum_{j \in \mathbb{N}} a_{s_{i}^{\prime}, j} R=\sum_{j=m_{i-1}}^{m_{i}-1} a_{s_{i}^{\prime}, j} R$ if $2 \leq i<k$. Moreover, $a_{i, j}=0$ for any $1 \leq j \leq m_{l}$ and $i \geq m_{l}^{\prime}$. This concludes the proof of the claim.

Now we can construct a sequence $p_{1}, p_{2}, \ldots$ of elements in $P$, $p_{i}=\left(c_{j, i}\right)_{j \in \mathrm{N}}$ say, such that there exist integers $1=i_{1}<i_{2}<\cdots$ with $I \subseteq R c_{i_{k}, k}+\cdots+R c_{i_{k+1}-1, k}$ for any $k \in \mathbb{N}$ and $c_{l, k}=0$ for any $l \geq i_{k+1}$. We proceed by induction again. Put $i_{1}=1$. By the claim, there exists $p_{1}$ such that $I \subseteq \sum_{j \in \mathrm{N}} R c_{j, 1}$. Of course, there exists $i_{2}>i_{1}$ with $c_{l, 1}=0$ for every
$l \geq i_{2}$.

Suppose we have $p_{1}, \ldots, p_{k}$ and $i_{1}, \ldots, i_{k+1}$. By the claim, there exists 
$p_{k+1}$ such that $I \subseteq \sum_{j \geq i_{k+1}} R c_{j, k+1}$. Let $i_{k+2}>i_{k+1}$ be an integer such that $c_{j, k+1}=0$ for all $j \geq i_{k+2}$.

Now, let $Q$ be a countably generated projective module with trace ideal contained in $I$ given by a column-finite idempotent matrix $B$ over $R$ (again, we consider $Q$ as a submodule of $\left.R^{(\mathrm{N})}\right)$. Since the trace ideal of $Q$ lies in $I$, all entries of $B$ are in $I$. Let $C$ be a matrix such that columns of $C$ are given by $p_{1}, p_{2}, \ldots$. The shape of $C$ guarantees the existence of a column-finite matrix $D$ having all entries in $\operatorname{Tr}(Q)$ such that $D C=B$ (it is important to realize that the elements of $D$ can be chosen in $I)$. Now, let $f: R^{(\mathrm{N})} \rightarrow R^{(\mathrm{N})}$ be given by $D$. Observe, that $Q \subseteq f(P)$ and that if $\pi: R^{(\mathrm{N})} \rightarrow Q$ is a projection, then $\left.\pi f\right|_{P}$ is an epimorphism of $P$ onto $Q$. Hence $P$ is $I$-big.

REMARK 2.9. Imitating the proof of [3, Theorem 3.1], we could get the following. Let $R$ be a ring such that $R / J(R)$ is right noetherian. Let $P, I_{k}$ be as above and suppose that $I=I_{n}=I_{n+1}=\cdots$ is a finitely generated left ideal such that $I \cap J(R)=J(R) I$. Then $P$ is $I$-big. (For $I=R$ we get Bass' big projectives theorem). Also we could omit the assumption (*) and prove that $P$ is $\cap_{n \in \mathbb{N}} I_{n}$-big. We do not give the details because we do not have applications for this version of Lemma 2.8.

Comparing the definition of $I_{0}$ in the proof of Lemma 2.4 and the statement of Lemma 2.8, we immediately get

COROLLARY 2.10. Let $R$ be a left and right noetherian ring satisfying (*). If $P$ is a countably generated projective $R$-module and $I$ is the least ideal of $R$ such that P/PI is finitely generated, then $P$ is I-big.

Obviously, Lemma 2.8 can be applied to study projective (right) modules over left and right noetherian rings satisfying $(*)$. The following lemma shows that over these rings we can apply Lemma 2.8 also for projective left modules. Recall that an $\mathbb{N} \times \mathbb{N}$ matrix $A=\left(a_{i, j}\right)_{i, j \in \mathbb{N}}$ is said to be row-finite if for any $i \in \mathbb{N}$ there exists $j \in \mathbb{N}$ such that $a_{i, k}=0$ for every $k \geq j$.

LEMma 2.11. Let $R$ be a left and right noetherian ring satisfying $\left(^{*}\right)$. Let $A=\left(a_{i, j}\right)_{i, j \in \mathrm{N}}$ be a row-finite matrix over $R$ such that $A^{2}=A$. For any $k \in \mathbb{N}$ let $I_{k}=\sum_{j>k, i \in \mathbb{N}} R a_{i, j} R$. Then there exists $n \in \mathbb{N}$ such that $I_{m}=I_{n}$ for
any $m \geq n$. 
Proof. Throughout the proof, we will work inside the left module $F={ }_{R} R^{(\mathrm{N})}$. Let $e_{1}, e_{2}, \ldots$ be the canonical free basis of $F$. For any $i \in \mathbb{N}$, let $a_{i}$ be the $i$-th row of $A$, that is, $a_{i}=\left(a_{i, 1}, a_{i, 2}, \ldots\right) \in F$. Thus $A$ gives a left projective module $P=F A=\sum_{i \in \mathbb{N}} R a_{i}$. For any $l \in \mathbb{N}_{0}$ let $\pi_{l}: F \rightarrow{ }_{R} R^{l}$ be the projection given by $\pi_{l}\left(\left(x_{1}, x_{2}, \ldots\right)\right)=\left(x_{1}, \ldots, x_{l}\right)$ (as usual, $R_{R} R^{0}$ is the trivial left $R$-module). For any $i \in \mathbb{N}$ let $c_{i}: F \rightarrow{ }_{R} R$ be the projection given by $c_{i}\left(\left(x_{1}, x_{2}, \ldots\right)\right)=x_{i}$.

Construct integers $0=n_{1}<n_{2}<\cdots$ and ideals $J_{1} \supseteq J_{2} \supseteq \cdots$ as follows: Put $n_{1}=0$ and $J_{1}=\sum_{i, j \in \mathbb{N}} R a_{i, j} R$. Suppose that $n_{k}$ and $J_{k}$ have been defined. Since $R$ is left noetherian, there exists $l \in \mathbb{N}$ such that the module $\pi_{n_{k}}(P)$ is generated by $\pi_{n_{k}}\left(a_{1}\right), \ldots, \pi_{n_{k}}\left(a_{l}\right)$. As $A$ is row-finite, there exists $m>n_{k}$ such that $a_{i, m^{\prime}}=0$ for any $1 \leq i \leq l, m^{\prime} \geq m$. Set $n_{k+1}=m$. Let $J_{k+1}$ be the ideal generated by $\{r \in R \mid$ there exist $p \in P$ and $i \in \mathbb{N}$ such that $\pi_{n_{k+1}}(p)=0$ and $\left.c_{i}(p)=r\right\}$. We claim that $J_{k+1} J_{k}=J_{k+1}$. In order to prove the claim, it suffices to prove that $S \subseteq J_{k+1} J_{k}$ for a set $S$ generating $J_{k+1}$. Let $p \in P$ be such that $\pi_{n_{k+1}}(p)=0$. Write $p=\left(0, \ldots, 0, r_{n_{k+1}+1}, \ldots\right)$. Then $p=r_{n_{k+1}+1}\left(e_{n_{k+1}+1} A\right)+r_{n_{k+1}+2}\left(e_{n_{k+1}+2} A\right)+\cdots$ From the construction it follows that for any $i \in \mathbb{N}$ there exists $p_{i} \in P$ such that $\pi_{n_{k}}\left(p_{i}\right)=0$ and $c_{n_{k+1}+j}\left(p_{i}\right)=c_{n_{k+1}+j}\left(e_{n_{k+1}+i} A\right)$ for every $j \in \mathbb{N}$. Since $c_{n_{k+1}+j}\left(p_{i}\right) \in J_{k}$, the equation

$r_{n_{k+1}+i}=c_{n_{k+1}+i}(p)=r_{n_{k+1}+1} c_{n_{k+1}+i}\left(\left(e_{n_{k+1}+1}\right) A\right)+r_{n_{k+1}+2} c_{n_{k+1}+i}\left(\left(e_{n_{k+1}+2}\right) A\right)+\cdots$ implies that $J_{k+1}=J_{k+1} J_{k}$.

As $R$ satisfies (*), there exists $m \in \mathbb{N}$ such that $J_{m}=J_{m+1}=\cdots$ Clearly, $J_{k} \subseteq I_{n_{k}}$ for any $k \in \mathbb{N}$. On the other hand, $I_{n_{k+1}} \subseteq J_{n_{k}}$. This concludes the proof of the lemma.

Let $R$ be a ring, let $V_{r}(R)$ be a set of representatives of the finitely generated projective right $R$-modules, $V_{l}(R)$ be a set of representatives of the finitely generated projective left $R$-modules, $V_{r}(R)^{*}$ be a set of representatives of the countably generated projective right modules and $V_{l}(R)^{*}$ be a set of representatives of the countably generated projective left $R$-modules. In the following theorem we consider $V_{r}(R / R)$ and $V_{l}(R / R)$ as sets containing one element.

THEOREM 2.12. Let $R$ be a left and right noetherian ring satisfying $(*)$. Let $\operatorname{Id}(R)$ be the set of its idempotent ideals and let $\mathcal{S}$ be the disjoint union $\dot{\cup}_{I \in \operatorname{Id}(R)} V_{r}(R / I)$. Then there is a bijection $\varphi: V_{r}(R)^{*} \rightarrow \mathcal{S}$. Moreover, there exists a bijection between $V_{r}(R)^{*}$ and $V_{l}(R)^{*}$ extending the classical bijection between $V_{r}(R)$ and $V_{l}(R)$ induced by $\operatorname{Hom}_{R}\left(-, R_{R}\right)$. 
Proof. By Corollary 2.10, any countably generated projective right module $P$ is $I$-big, where $I$ is the least ideal such that $P / P I$ is finitely generated. We know that $I$ is idempotent. This gives a map of $V_{r}(R)^{*}$ into $\mathcal{S}$. This map is a bijection by Lemmas 2.5 and 2.6.

Of course, all the results of this section can be formulated for left modules. We do not know whether condition (*) is equivalent to condition (*'): Let $I_{1}, I_{2}, \ldots$ be a sequence of ideals such that $I_{k} I_{k+1}=I_{k+1}$ for any $k \in \mathbb{N}$. Then there exists $n \in \mathbb{N}$ such that $I_{n}=I_{n+1}=\ldots$ Condition $(*)$ is connected to right modules while (*') is connected to left modules. Therefore it would be more precise to talk about condition right (*) instead of (*). In order to be concise, we have omitted the word "right", but the reader should be aware that this condition has to be changed formulating the versions of the results for left modules. However, we can use Lemma 2.11 and the "left version" of Lemma 2.8 to see that any countably generated projective left module $Q$ is $I$-big, where $I$ is the least ideal such that $Q / I Q$ is finitely generated. Again, $I$ is idempotent and finitely generated as a right module, therefore the "left versions" of Lemma 2.5 and Lemma 2.6 give a bijection of $V_{l}^{*}(R)$ and the disjoint union $\dot{\cup}_{I \in \operatorname{Id}(R)} V_{l}(R / I)$. The bijection between $V_{r}^{*}(R)$ and $V_{l}^{*}(R)$, then follows from the dualities between finitely generated projective left and right $R / I$-modules, where $I$ varies in $\operatorname{Id}(R)$.

REMARK 2.13. Observe that if $R$ is a left and right noetherian ring having (*), then every indecomposable projective module is finitely generated. Although we think that (*) is a very particular property (see [8] for examples of rings having infinite properly descending chains of idempotent ideals), it seems to occur quite often in natural examples of left and right noetherian rings.

\section{Semilocal noetherian rings.}

Recall that a ring $R$ is said to be semilocal, if the factor of $R$ modulo its Jacobson radical is semisimple artinian. Throughout the paper, $J(R)$ denotes the Jacobson radical of $R$. If $P, Q$ are projective modules, then $P / P J(R) \simeq Q / Q J(R)$ if and only if $P \simeq Q$ [13, Theorem 1.3]. In this section, we show that semilocal left and right noetherian rings satisfy (*), so that any countably generated projective module over such a ring is fair-sized. Further, we show a connection between the pair $(I, P / P I)$ defined in the previous section and the semisimple module $P / P J(R)$. Finally, we give an 
example of superdecomposable projective module over a semilocal noetherian ring.

Recall that if $P$ is a projective module over $R$, then the intersection of all maximal submodules of $P$, called the radical of $P$, is $\operatorname{rad}(P)=P J(R)$. If $R$ is semilocal and $S_{1}, \ldots, S_{k}$ are representatives of the simple $R$-modules (that is, for any simple $R$-module $S$ there exists exactly one $i \in\{1, \ldots, k\}$ with $S \simeq S_{i}$ ), then for every projective module $P$ there are cardinals $\lambda_{1}, \ldots, \lambda_{k}$, uniquely determined, such that $P / P J(R)=S_{1}^{\left(\lambda_{1}\right)} \oplus \cdots \oplus S_{k}^{\left(\lambda_{k}\right)}$. We will write $\operatorname{dim}(P)=\left(\lambda_{1}, \ldots, \lambda_{k}\right)$. Clearly, $\operatorname{dim}$ depends on the ordering of the representatives of the simple $R$-modules. Therefore we will always suppose that with any semilocal ring $R$ we have some fixed ordering on the set of representatives of the simple $R$-modules. By [13, Theorem 1.3], two projective $R$-modules $P$ and $Q$ are isomorphic if and only if $\operatorname{dim}(P)=\operatorname{dim}(Q)$.

LEMMA 3.1. Let $R$ be a right noetherian semilocal ring. If I and $K$ are idempotent ideals of $R$ such that $I+J(R)=K+J(R)$, then $I=K$. In particular, $R$ has only finitely many idempotent ideals.

Proof. Since $R / J(R)$ has only finitely many (idempotent) ideals, it is enough to show that $I+J(R)=K+J(R)$ implies $I=K$ whenever $I$ and $K$ are idempotent ideals of $R$.

First suppose that $I \subseteq K$ are idempotent ideals of $R$. In particular, $K I=I$. Suppose that $I+J(R)=K+J(R)$. Then $K=K(K+J(R))$ $=K(I+J(R))=I+K J(R)$. Since $R$ is right noetherian, Nakayama's Lemma gives $I=K$.

In general, suppose that $I$ and $K$ are idempotent ideals of $R$ with $I+J(R)=K+J(R)$. Then $I$ and $I+K$ are idempotent ideals of $R$ such that $I+J(R)=I+K+J(R)$. By the previous step, $I=I+K$, and therefore $K \subseteq I$. The proof for $I \subseteq K$ is similar.

COROLlary 3.2. Let $R$ be a right noetherian semilocal ring. Then $R$ satisfies condition (*).

Proof. Let $\pi: R \rightarrow R / J(R)$ be the natural projection. Consider a descending sequence of ideals in $R$ such that $I_{k+1} I_{k}=I_{k+1}$. Since $\pi\left(I_{1}\right), \pi\left(I_{2}\right), \ldots$ is a descending sequence in an artinian ring $R / J(R)$, there exists $k_{0} \in \mathbb{N}$ such that $\pi\left(I_{k}\right)=\pi\left(I_{k_{0}}\right)$ for every $k \geq k_{0}$. Then $I_{k+1}$ $=I_{k+1}\left(I_{k+1}+J(R)\right)=I_{k+1}^{2}+I_{k+1} J(R)$ for every $k \geq k_{0}$. By Nakayama's Lemma, we see that $I_{k}$ is idempotent for any $k>k_{0}$. Now conclude by Lemma 3.1. 
The following lemma and its application was suggested by Dolors Herbera.

Lemma 3.3. Let $P$ be a projective $R$-module with trace ideal I and let $S$ be a simple $R$-module. The following conditions are equivalent.

(i) $S$ is a factor of $I_{R}$.

(ii) $S$ is a factor of $P$.

(iii) $S I=S$.

Proof. (i) $\Rightarrow$ (ii) Suppose that $f: I \rightarrow S$ is nonzero. Then $f(i) \neq 0$ for some $i \in I$. Since $I$ is the trace ideal of $P$, there are homomorphisms $g_{1}, \ldots, g_{k}: P \rightarrow I$ and $p_{1}, \ldots, p_{k} \in P$ with $g_{1}\left(p_{1}\right)+\cdots+g_{k}\left(p_{k}\right)=i$. Therefore $f g_{j} \neq 0$ for some $1 \leq j \leq k$. (Observe that we did not use $P$ projective for this implication.)

(ii) $\Rightarrow$ (iii) Follows from $P I=P$.

(iii) $\Rightarrow$ (i) Let $f: R_{R} \rightarrow S$ be nonzero. Then $f(I)=S$, because $S I=S$.

Proposition 3.4. Let $R$ be a semilocal left and right noetherian ring. Suppose that $P$ is a countably generated projective module. Then there exists a least ideal I in $R$ such that P/PI is finitely generated.

Moreover, let $\left\{S_{1}, \ldots, S_{k}\right\}$ be a set of representatives of the simple modules, indexed in such a way that $P / P J(R) \simeq S_{1}^{n_{1}} \oplus \cdots \oplus S_{l}^{n_{l}} \oplus$ $\oplus S_{l+1}^{(\omega)} \oplus \cdots \oplus S_{k}^{(\omega)}, n_{1}, \ldots, n_{l} \in \mathbb{N}_{0}, 0 \leq l \leq k$. Then:

(i) $P$ is I-big,

(ii) $S_{i}=S_{i} I$ if and only if $i>l$,

(iii) $P / P I / \operatorname{rad}(P / P I) \simeq S_{1}^{n_{1}} \oplus \cdots \oplus S_{l}^{n_{l}}$.

Proof. We have seen in Corollary 3.2 that $R$ satisfies (*). By Lemma 2.4, there exists $I$ such that $P / P I$ is finitely generated and $I$ is contained in any other ideal $K$ such that $P / P K$ is finitely generated. Moreover, $P$ is $I$-big according to Corollary 2.10. Since $I$ is finitely generated as a left ideal, there exists a unique $I$-big projective module $B$ with trace ideal $I$ and $P \oplus B^{(\omega)} \simeq P$ according to Remark 2.1. By Lemma 3.3 , if $S$ is a simple module, then $S^{(\omega)}$ is a factor of $P$ (and hence of $P / P J(R)$ ) whenever $S I=S$. Choose an enumeration of the simple modules such that $S_{1}, \ldots, S_{l}$ are annihilated by $I$ and $S_{l+1}, \ldots, S_{k}$ are factors of $I$. Let $0 \leq \lambda_{1}, \ldots, \lambda_{k} \leq \infty$ be such that $P / P J(R) \simeq S_{1}^{\left(\lambda_{1}\right)} \oplus \cdots \oplus S_{k}^{\left(\lambda_{k}\right)}$. As remarked above, $\lambda_{l+1}=\cdots=\lambda_{k}=\infty$. On the other hand, $S_{1}^{\left(\lambda_{1}\right)} \oplus \cdots \oplus S_{l}^{\left(\lambda_{l}\right)}$ is a factor of $P$ annihilated by $I$, hence a factor of $P / P I$. 
Thus $\lambda_{1}, \ldots, \lambda_{l}$ are finite. Suppose $P / P I / \operatorname{rad}(P / P I) \simeq S_{1}^{n_{1}} \oplus \cdots \oplus S_{l}^{n_{l}}$. Since $S_{1}^{\lambda_{1}} \oplus \cdots \oplus S_{l}^{\lambda_{l}}$ is a semisimple factor of $P / P I, \lambda_{i} \leq n_{i}$ for any $1 \leq i \leq l$. On the other hand, $S_{1}^{n_{1}} \oplus \cdots \oplus S_{l}^{n_{l}}$ is a factor of $P$, so that $n_{i} \leq \lambda_{i}$ for every $1 \leq i \leq l$.

Recall that a nonzero module is called superdecomposable if it has no indecomposable direct summand. The following lemma explains our craving for the existence of superdecomposable projectives over semilocal rings.

Lemma 3.5. Suppose that there exists a superdecomposable projective module over a semilocal ring $R$. Then $R$ possesses a nonzero decomposable projective module having all its nonzero direct summands isomorphic.

Proof. By the theorem of Kaplansky, if there exists a superdecomposable projective module, then there exists a superdecomposable countably generated projective module. It follows easily that then there exists a superdecomposable countably generated projective module $Q$ such that $\operatorname{dim}(Q)=\left(m_{1}, \ldots, m_{k}\right)$, where $m_{i}=0$ or $m_{i}=\omega$ for any $1 \leq i \leq k$ (use the additivity of dim). Let $Q^{\prime}$ be a superdecomposable module such that $\operatorname{dim}\left(Q^{\prime}\right)$ has all components in $\{0, \omega\}$ and the number of nonzero components is as small as possible. Then it is easy to see that $\operatorname{dim}\left(Q^{\prime}\right)=\operatorname{dim}\left(Q^{\prime \prime}\right)$ for any nonzero direct summand of $Q^{\prime}$, so [13, Theorem 1.3] gives that $Q^{\prime}$ has the required property.

The following example discovered by Puninski [12] shows that a superdecomposable projective module may exist even over a semilocal noetherian ring.

Example 3.6 (cf. [12, Proposition 7.5]). Let $\Sigma=Z \backslash 2 Z \cup 3 Z \cup 5 Z$ and let $Z_{\Sigma}$ be the localization of integers at $\Sigma$. Let $A_{5}$ be the group of even permutations on the set of cardinality 5 . Then the group ring $Z_{\Sigma}\left[A_{5}\right]$ is a semilocal left and right noetherian ring with a superdecomposable projective module.

Proof. We will repeat general arguments of [12] that show that the ring $R=Z_{\Sigma}\left[A_{5}\right]$ is a semilocal left and right noetherian ring. First, $R$ is a finitely generated as a (left and right) module over the commutative noetherian ring $Z_{\Sigma}$, therefore $R$ is noetherian on both sides. Further, $R \simeq \operatorname{End}_{R}\left(R_{R}\right)$, so that there exists an injective homomorphism $\varphi: R \rightarrow \operatorname{End}_{Z_{\Sigma}}(R)$ given by the left 
multiplication of $R$ on $R_{Z_{\Sigma}}$. For any $g \in A_{5}$, let $\theta_{g} \in \operatorname{End}_{Z_{\Sigma}}(R)$ be given by $\theta_{g}(r)=r g, r \in R$. Obviously, $\operatorname{Im} \varphi$ consists exactly of the elements of $\operatorname{End}_{Z_{\Sigma}}(R)$ that commute with all the endomorphisms of the set $\left\{\theta_{g} \mid g \in A_{5}\right\}$. It follows that $\varphi$ is a local homomorphism, that is, $r$ is invertible in $R$ if $\varphi(r)$ is invertible in $\operatorname{End}_{Z_{\Sigma}}(R)$. Finally, since $\operatorname{End}_{Z_{\Sigma}}(R) \simeq \mathrm{M}_{60}\left(Z_{\Sigma}\right)$ is a semilocal ring, the ring $R$ is also semilocal by [4, Theorem 1$]$.

Let $I$ be the augmentation ideal of $R$, that is, the kernel of the epi$\operatorname{morphism} f: R \rightarrow Z_{\Sigma}, f\left(\sum_{g \in A_{5}} r_{g} g\right)=\sum_{g \in A_{5}} r_{g}$. Since $\left[A_{5}, A_{5}\right]=A_{5}$, the ideal $I$ is idempotent [1, Theorem 2.1]. By [12], it can be proved that every nonzero finitely generated projective module over $R$ is a generator. In fact, we only need to show that if $P$ is a finitely generated projective $R$-module, then $\operatorname{Tr}(P)$ cannot be contained in $I$ : Since $Z_{\Sigma}$ is a Dedekind ring of zero characteristic and 2,3,5 are not invertible in $Z_{\Sigma}, P^{\prime}=P \otimes_{Z_{\Sigma}\left[A_{5}\right]} \mathrm{Q}\left[A_{5}\right]$ is a free $\mathrm{Q}\left[A_{5}\right]$-module by [16, Theorem 8.1]. If $\operatorname{Tr}(P) \subseteq I$, then $P^{\prime} I^{\prime}=P^{\prime}$, where $I^{\prime}$ is the augmentation ideal of $\mathrm{Q}\left[A_{5}\right]$, a contradiction. Let $Q$ be a projective module having trace ideal $I$. If $Q^{\prime}$ is a nonzero direct summand of $Q$, then $Q^{\prime}$ cannot be finitely generated, and there is a nonzero idempotent ideal $K$ such that $Q^{\prime}$ is $K$-big. Therefore $Q^{\prime}$ cannot be indecomposable.

REMARK 3.7. In the next section we look closer at the localizations of $Z\left[A_{5}\right]$ showing that the augmentation ideal of $Z_{\Sigma}\left[A_{5}\right]$ contains no nontrivial idempotent ideals.

\section{Integral group rings, especially $Z\left[A_{5}\right]$.}

In this section, we prove that an integral group ring of a finite group satisfies condition (*). The proof presented here is not the quickest one, but it shows how to calculate idempotent ideals in particular examples. We apply this method to $Z\left[A_{5}\right]$ describing all countably but not finitely generated projective modules up to isomorphism. Our approach will be elementary.

First of all, let us introduce the notation we will use throughout this section. Let $G$ be a finite group and $R=Z_{[}[G], R_{p}=Z_{(p)}[G], R_{0}=\mathrm{Q}[G]$. For any prime $p$ we have $R \subseteq R_{p} \subseteq R_{0}$. If $I$ is an ideal of $R, I_{(p)}$ stands for the ideal in $R_{p}$ generated by $I$ and $I_{(0)}$ stands for the ideal of $R_{0}$ generated by $I$. That is, $I_{(p)}=Z_{(p)} I$ and $I_{(0)}=Q I$. We say that an ideal $I \subseteq R$ (or an ideal $I \subseteq R_{p}$ ) extends to an ideal $K \subseteq R_{0}$ if $K=\mathrm{Q} I$. If $S$ is a commutative ring, the augmentation ideal of $S[G]$ is the kernel of the canonical 

$\operatorname{homomorphism} f: S[G] \rightarrow S$ given by $f\left(\sum_{g \in G} s_{g} g\right)=\sum_{g \in G} s_{g}$. It is denoted by
$\operatorname{Aug}(S[G])$.

In the following we summarize the framework of our calculations.

FACT 4.1. Let $G$ be a finite group and let $R=Z[G]$. Then

(i) If $I$ is an ideal of $R$, then $I_{(0)}=Q I_{(p)}$ for every prime $p$.

(ii) Let $I, K$ be ideals in $R$. Then $I=K$ if and only if $I_{(p)}=K_{(p)}$ for every prime $p$.

(iii) If $I \subseteq R$ is an ideal, then $I$ is idempotent if and only if $I_{(p)}$ is idempotent for every prime $p$.

(iv) If $I, K$ are idempotent ideals of $R$ and $p$ a prime not dividing $|G|$, then $I_{(p)}=K_{(p)}$ if and only if $I_{(0)}=K_{(0)}$. In this case, all central idempotents of $R_{0}$ are contained in $R_{p}$ and every idempotent ideal of $R_{p}$ is generated by a central idempotent.

(v) Let e be a central idempotent of $R_{0}$ and suppose that, for every prime $p$ that divides $|G|$, there is an idempotent ideal $I_{p} \subseteq R_{p}$ with $\mathrm{Q} I_{p}=e R_{0}$. Then there exists a unique idempotent ideal $I \subseteq R$ such that $I_{(p)}=I_{p}$ for any $p|| G \mid$ and $I_{(p)}=e R_{p}$ for any $p \nmid|G|$.

Proof. Statements (i), (ii), (iii) and (v) are rather standard. Statement (iv) follows from the fact that $Z_{(p)}[G]$ is a maximal $Z_{(p)}$-order in $\mathrm{Q}[G]$ if and only if $p$ does not divide $|G|$ (see [5, Proposition 27.1]) and using the machinery of maximal orders.

Here we give another proof of (iv). Let $Q \subseteq F$ be a finite Galois extension of $Q$ such that $F$ is a splitting field of $G$. Recall that if $\xi$ is a complex character of a simple representation of $G$ over $F$ (considered as a function $\xi: G \rightarrow F)$, then $\frac{\xi\left(1_{G}\right)}{|G|}\left(\sum_{g \in G} \xi\left(g^{-1}\right) g\right)$ is a primitive central idempotent of $F[G]$. In order to get the set of primitive central idempotent of $Q[G]$, consider the usual action of $\operatorname{Gal}(F: Q)$ on the set of primitive central idempotents of $F[G]$ and take sums of the orbits. It follows that if $p$ is a prime and $p \nmid|G|$, then any central idempotent of $R_{0}$ is in $R_{p}$.

Let $I$ be an idempotent ideal of $R_{p}$, where $p$ is a prime not dividing $|G|$. Then $\mathrm{Q} I$ is an ideal of $R_{0}$ generated by a central idempotent $e$ of $R_{0}$. Since $e \in R_{p}, K=e R_{p}$ is an idempotent ideal of $R_{p}$, necessarily $I \subseteq K$ because $e I=I$. Since $\mathrm{Q} I=\mathrm{Q} K$, there exists $k \in \mathbb{N}$ such that $p^{k} K \subseteq I$. As $Z_{p}[G]$ is semisimple, idempotent ideals in $Z_{p^{n}}[G]$ are generated by central idempotents for any $n \in \mathbb{N}$ (combine [2, Proposition 27.1] and [10,22.10]). Moreover, it is easily seen that if $K^{\prime}$ is an idempotent ideal of $Z_{p^{2 n}}[G]$, then $p^{n} K^{\prime}$ is an 
essential submodule of $K^{\prime}$. Now let $\pi: R_{p} \rightarrow Z_{p^{2 k}}[G]$ be the canonical projection. Then $p^{k} \pi(K) \subseteq \pi(I) \subseteq \pi(K)$. By our previous remarks, $\pi(I)=\pi(K)$. Since $R_{p}$ is a semilocal noetherian ring and $\pi$ is an epimorphism with Ker $\pi \subseteq J\left(R_{p}\right)$ (Fact 4.3), $I=K$ follows from Lemma 3.1.

The following result also follows from [15, Theorem 3].

COROLlary 4.2. Any integral group ring of a finite group satisfies (*) and has only finitely many idempotent ideals.

Proof. Since $R$ is a ring of Krull dimension 1 , it is enough to see that $R$ has no descending chain of idempotent ideals. Let $I$ be an idempotent ideal, let $e$ be a central idempotent of $R_{0}$ such that $e R_{0}=Q I$. Then $I_{(p)}=e R_{p}$ for every prime $p$ not dividing $|G|$ by Fact 4.1(iv). If $p$ is a prime divisor of $|G|$, then we have only finitely many possibilities for $I_{(p)}$ by Lemma 3.1. Therefore, by Fact 4.1(v), $R$ contains only finitely many idempotent ideals.

The proof of Corollary 4.2 shows a method of finding idempotent ideals in $R$. We can proceed as follows: Take an ideal $I_{0}$ of $R_{0}$. Let $P$ be the set of prime divisors of $|G|$. For any $p \in P$, determine the set $M_{p}$ consisting of the idempotent ideals of $R_{p}$ that extend to $I_{0}$. Then there is a bijective correspondence between the idempotent ideals of $R$ extending to $I_{0}$ and the set $\prod_{p \in P} M_{p}$.

Thus we can now work in semilocal localizations (see [5] or use the same kind of arguments as in Example 3.6).

FACT 4.3. The natural homomorphism $\pi_{p}: R_{p} \rightarrow Z_{p}[G]$ is a local morphism for any prime $p$. In particular, $p R_{p} \subseteq J\left(R_{p}\right)$ and $R_{p}$ is a semilocal ring.

Let us show the method in the case of $G=A_{5}$, the alternating group on 5 elements. The usual question "Why $A_{5}$ ?" has a simple answer. By a result of Swan [17], non-finitely generated projective modules over integral group rings of finite solvable groups are free. Therefore there are no proper idempotent ideals in integral group rings of finite solvable groups (a direct proof of this was given by Roggenkamp [14]). On the other hand, it is known [1] that if $G$ contains a perfect normal subgroup $H$, that is, $[H, H]=H$ and $H \unlhd G$, then the augmentation ideal of $H$ (that is, the kernel 
of the canonical homomorphism $Z[G] \rightarrow Z[G / H]$ ) is idempotent. If there were no other idempotent ideals in $Z[G]$, then countably generated projective modules over $Z[G]$ would be induced by finitely generated projective modules over $Z[G / H]$, where $H$ ranges in the set of perfect normal subgroups of $G$. So $A_{5}$ is the first candidate to check. Unfortunately, we will see that there indeed exists an idempotent ideal that is not the augmentation ideal of a perfect normal subgroup. Hence the structure theory for big projective modules over integral group rings seems to be more complicated.

Throughout the next paragraphs, suppose $G=A_{5}$. The conjugacy classes of $G$ are the following: $c_{1}$ - the conjugacy class of the identity; $c_{2}$ the permutations that are product of two 2-cycles (the conjugacy class of $(1,2)(3,4)) ; c_{3}$ - all 3 -cycles; $c_{5}$ - the conjugacy class of $(1,2,3,4,5)$; and $c_{5}^{\prime}$ the conjugacy class of $(1,3,5,2,4)$.

Let us recall what we know about the semisimple ring $R_{0}$. The primitive central idempotents of $R_{0}$ are $e_{1}=\frac{1}{60} \sum_{g \in G} g, e_{3}=\frac{1}{20}\left(6-2 \sum_{g \in c_{2}} g+\sum_{g \in c_{5} \cup c_{5}^{\prime}} g\right)$, $e_{2}=\frac{1}{15}\left(4+\sum_{g \in c_{3}} g-\sum_{g \in c_{5} \cup c_{5}^{\prime}} g\right), e_{5}=\frac{1}{12}\left(5+\sum_{g \in c_{2}} g-\sum_{g \in c_{3}} g\right)$. Let $T_{1}, T_{3}, T_{2}, T_{5}$ be the corresponding simple modules $\left(e_{i}\right.$ corresponds to $\left.T_{i}\right)$. Their dimensions over $Q$ are $1,6,4,5$.

We need to calculate the idempotent ideals in $R_{2}, R_{3}, R_{5}$. Set $S_{i}=Z_{i}\left[A_{5}\right]$ for $i=2,3,5$. By Fact 4.3 , any simple $S_{i}$-module can be considered as a simple $R_{i}$-module and there are no other simple $R_{i}$-modules except for these. In order to find the number of different simple modules over $R_{i}$, one can use the following results proved by Berman and Witt (see [5, Theorem 21.5, Theorem 21.25]).

FACT 4.4. Let $G$ be a finite group of exponent $m$.

(i) Let $\sim$ be the relation on $G$ given by $g \sim h$ if $g$ is conjugate to $h^{t}$ for some $t \in \mathbb{N},(t, m)=1$. Then the number of simple $\mathrm{Q}[G]$-modules is equal to $|G / \sim|$.

(ii) Let $p$ be a prime, and $G_{p^{\prime}}$ the set of p-regular elements of $G$. On the set $G_{p^{\prime}}$ consider the equivalence $\sim$ defined by $g \sim h$ if $g$ is conjugate to $h^{p^{j}}$ for some $j \in \mathbb{N}_{0}$. Then the number of simple $Z_{p}[G]$-modules is equal to $\left|G_{p^{\prime}} / \sim\right|$.

Thus each ring $R_{2}, R_{3}, R_{5}$ has exactly three non-isomorphic simple modules. Now idempotent ideals in semilocal rings are determined by their 
simple factors (Lemma 3.1). Call a ring $T$ almost semiperfect if for any simple $T$-module $M$ there exists a positive integer $n$ such that $M^{n}$ has a projective cover. The next lemma describes the distribution of idempotent ideals in $R_{i}$, for $i \in\{2,3,5\}$. In all the remaining proofs of this section, $I_{i}$ stands for $\operatorname{Aug}\left(R_{i}\right)$.

LEMma 4.5. Let $i \in\{2,3,5\}$. The ring $R_{i}$ has exactly 3 minimal idempotent ideals and any nonzero idempotent ideal of $R_{i}$ is a sum of minimal idempotent ideals. Moreover, $R_{i}$ is almost semiperfect and any idempotent ideal of $R_{i}$ is a trace ideal of a finitely generated projective module. Finally, two minimal idempotent ideals are described as follows: If $I_{i}$ is the augmentation ideal of $R_{i}$, then $e_{i} R_{i}$ and $\left(1-e_{i}\right) I_{i}$ are minimal idempotent ideals of $R_{i}$.

Proof. We give the proof for $i=5$, the remaining cases are similar. The augmentation ideal $I_{5} \subseteq R_{5}$ is idempotent, because $A_{5}$ is perfect. Observe $e_{5} \in R_{5}$. Therefore also $e_{5} R_{5}$ and $\left(1-e_{5}\right) I_{5}$ are idempotent ideals. Let $M_{1}, M_{2}, M_{3}$ be the set of representatives of the simple $R_{5}$-modules and suppose that $M_{1}$ is the module induced by the trivial representation of $S_{5}$. Obviously, $M_{1} I_{5}=0$, so $M_{1}$ is not a factor of $I_{5}$. Since $I_{5}$ must have at least two simple factors (it contains two different nontrivial idempotent ideals), $M_{2}, M_{3}$ are both factors of $I_{5}$. Choose the notation in such a way that $M_{2}$ is the unique simple factor of $\left(1-e_{5}\right) I_{5}$ and $M_{3}$ is the unique simple factor of $e_{5} R_{5}$.

Obviously, $e_{5} R_{5}$ is the trace ideal of the projective module $e_{5} R_{5}$. Set $g=(1,2)(3,4)$. The idempotent $e^{\prime}=\left(1-e_{5}\right)\left(1-\frac{1}{2}(1+g)\right)$ gives a projective $R_{5}$-module $P^{\prime}=e^{\prime} R_{5}$ with trace ideal $\left(1-e_{5}\right) I_{5}$. It follows that $P^{\prime} / P^{\prime} J\left(R_{5}\right)=M_{2}^{k}$, for some $k \in \mathbb{N}$ (it is necessary to check that $P^{\prime} \neq 0$, below we calculate $Z_{(5)}$-rank of $P^{\prime}$ using the so called Hattori-Stallings map).

On the other hand, the projective module $P=\left(1-e_{5}\right) R_{5}$ has the radical factor $P / P J\left(R_{5}\right)=M_{1} \oplus M_{2}^{l}$. Therefore $P^{\prime l}$ splits in $P^{k}$, that is, there exists a projective module $Q$ such that $P^{k}=P^{\prime l} \oplus Q$. Since $Q / Q J\left(R_{5}\right) \simeq M_{1}^{k}$, it follows that $\operatorname{Tr}(Q)$ is an idempotent ideal such that $M_{1}$ is its only simple factor.

So we have proved that the finitely generated projective modules $Q, P^{\prime}, e_{5} R_{5}$ are the projective covers of convenient finite powers of $M_{1}, M_{2}, M_{3}$ and $R_{5}$ is almost semiperfect. Therefore $\operatorname{Tr}(Q), \operatorname{Tr}\left(P^{\prime}\right)$ and $\operatorname{Tr}\left(e_{5} R_{5}\right)$ are the minimal idempotent ideals of $R_{5}$ and any nonzero idempotent ideal of $R_{5}$ is a sum of minimal idempotent ideals. 
LEMma 4.6. The only idempotent ideals of $R=Z\left[A_{5}\right]$ contained in $\operatorname{Aug}(R)$ are 0 and $\operatorname{Aug}(R)$.

Proof. Set $I=\operatorname{Aug}(R)$ and let $0 \neq K$ be an idempotent ideal of $R$ contained in $I$. Then $K_{(i)}$ also is a non-zero idempotent ideal of $R_{i}$ contained in $I_{i}$, hence, by Lemma 4.5, $\mathrm{Q} K_{(i)}$ is either $e_{i} R_{0},\left(e_{2}+e_{3}+e_{5}-e_{i}\right) R_{0}$ or $I_{(0)}=\left(e_{2}+e_{3}+e_{5}\right) R_{0}$. Now $\mathrm{Q} K_{(2)}=\mathrm{Q} K_{(3)}=\mathrm{Q} K_{(5)}=\mathrm{Q} K$. An easy inspection gives that the only possibility is $K_{(i)}=I_{i}$ for any $i \in\{2,3,5\}$. Therefore $K=I$ by Fact 4.1(v).

For any $i \in\{2,3,5\}$, let $K_{i}$ be the (unique) minimal idempotent ideal of $R_{i}$ that is not contained in the augmentation ideal of $R_{i}$. In order to classify the idempotent ideals in $R$ that are not contained in the augmentation ideal of $R$, we must determine $Q K_{2}, Q K_{3}$ and $Q K_{5}$. Let us prove an auxiliary general result, which is probably well known.

LEMMA 4.7. Let $\varphi: S \rightarrow T$ be a ring homomorphism. If $P$ is a projective $S$-module with trace ideal $I$, then $P \otimes_{S} T$ is a projective T-module with trace ideal $T \varphi(I) T$.

Proof. Let $X$ be a set and let $\pi: S^{(X)} \rightarrow S^{(X)}$ be an idempotent endomorphism of $S^{(X)}$ such that $\pi\left(S^{(X)}\right) \simeq P$. If $\pi$ is expressed as a columnfinite idempotent matrix $A$ (with respect to the canonical basis), then $\varphi(A)$ is an idempotent matrix corresponding to the endomorphism $\pi^{\prime}: T^{(X)} \rightarrow T^{(X)}$ such that $P \otimes_{S} T \simeq \pi^{\prime}\left(T^{(X)}\right)$. Now $\operatorname{Tr}(P)$ (resp. $\operatorname{Tr}\left(P \otimes_{S} T\right)$ ) is the ideal generated by the entries of $A$ (resp. $\varphi(A)$ ).

FACT 4.8. Let $S$ be a commutative local ring and let $H$ be a finite group. Suppose that $e=\sum_{h \in H} s_{h} h$ is an idempotent of $S[H]$. The module $e S[H]$ is free when considered as an $S$-module. Moreover, $|H| s_{1}=n \cdot 1_{S}$, where $n \in \mathbb{N}_{0}$ is the rank of the free $S$-module eS[H].

Proof. This is a consequence of [7, Example 7]. Let us briefly explain the idea. Let $T$ be a ring and $T /[T, T]$ be the group that is the factor of the additive group of $T$ modulo $[T, T]=\left\langle\left\{t_{1} t_{2}-t_{2} t_{1} \mid t_{1}, t_{2} \in T\right\}\right\rangle_{(T,+)}$. There exists a map $r: K_{0}(T) \rightarrow T /[T, T]$ defined as follows. Let $P$ be a finitely generated projective module over $T$ and $A$ an idempotent matrix representing $P$. Then $r([P]):=\operatorname{Tr}(A)+[T, T]$ (here $\operatorname{Tr}(A)$ is the sum of the diagonal entries in $A$ ). 
Since $S$ is a local ring, $K_{0}(S) \simeq Z$. As $S$ is commutative, $r$ is a well defined map of $K_{0}(S)$ into $S$. It follows that $\operatorname{Im} r \subseteq Z 1_{S}$. Now view $S[H]$ as a free $S$-module of rank $|H|$. The left multiplication by $e$ gives an idempotent endomorphism $\alpha$ of this $S$-module whose image is $e S[H]$. Now compute $r([e S[H]])$. Consider the matrix of $\alpha$ with respect to the basis $\{h \mid h \in H\}$. All the diagonal entries of this matrix are equal to $s_{1}$. Therefore $|H| \cdot s_{1}=n \cdot 1_{S}$, where $n$ is the rank of the free $S$-module $e S[H]$.

Now we can continue in $Z\left[A_{5}\right]$. In the following proofs $I_{i}$ is again the augmentation ideal of $R_{i}$ and $S_{i}=Z_{i}\left[A_{5}\right]$ for every $i \in\{2,3,5\}$.

LEMMA 4.9. Let $K_{5}$ be the minimal idempotent ideal not contained in $\operatorname{Aug}\left(R_{5}\right)$. Then $Q K_{5}=\left(e_{1}+e_{2}\right) R_{0}$.

Proof. Let $M_{1}, M_{2}, M_{3}$ be the simple $R_{5}$-modules such that $M_{1}$ is a unique simple factor of $K_{5}, M_{2}$ is a unique simple factor of $\left(1-e_{5}\right) I_{5}$ and $M_{3}$ is a unique simple factor of $e_{5} R_{5}$. Let $g=(1,2)(3,4)$. Then $e^{\prime}=\left(1-e_{5}\right)\left(1-\frac{1}{2}(1+g)\right)$ gives a projective $R_{5}$-module $P^{\prime}=e^{\prime} R_{5}$ with trace ideal $\left(1-e_{5}\right) I_{5}$, so it follows that $P^{\prime} / P^{\prime} J\left(R_{5}\right)=M_{2}^{k}$ for some $k \in \mathbb{N}$. Moreover, if $P=\left(1-e_{5}\right) R_{5}$, then $P / P J\left(R_{5}\right) \simeq M_{1} \oplus M_{2}^{l}$ for some $l \in \mathbb{N}$. We want to determine $k$ and $l$. The integer $l$ is given by the multiplicity of $M_{2}$ in $S_{5} / J\left(S_{5}\right)$. Any simple $S_{5}$-module is absolutely simple, therefore $l$ is equal to the dimension of the non-trivial simple representation that is annihilated by $e_{5}$. By [18, page 201], $l=3$. Obviously, $P^{\prime}$ is a direct summand of $P$, and $k \in\{1,2,3\}$ follows. Using Fact 4.8 , we have that the $Z_{(5)^{-}}$ rank of $P$ is equal to 35 and the $Z_{(5)}$-rank of $P^{\prime}$ is equal to 20 . If $k=1$, then $P^{\prime 3}$ would be a direct summand of $P$, which is not possible. Further, consider the $S_{5}$-module $P^{\prime} / P^{\prime} 5 R_{5}$. This is a vector space over $Z_{5}$ of dimension 20 . If $k=3$, then $P^{\prime} / P^{\prime} 5 R_{5} \simeq M^{3}$, where $M$ is an $S_{5}$-module which is a projective cover of $M_{2}$ if $M_{2}$ is considered as a simple $S_{5}$-module. Since 3 does not divide 20, this is also impossible. Therefore $k=2$.

As we have shown in the proof of Lemma $4.5, K_{5}$ is the trace ideal of $Q$, where $Q$ is a projective module defined by the relation $Q \oplus P^{\prime 3} \simeq P^{2}$. By Lemma 4.7, $Q K_{5}=\operatorname{Tr}\left(Q \otimes_{R_{5}} R_{0}\right)$. The module $Q \otimes_{R_{5}} R_{0}$ has Q-dimension 10 and contains the trivial representation of $R_{0}$ with multiplicity 2 . The only possibility (looking at the Q-dimension of the simple $R_{0}$-modules) is $Q \otimes_{R_{5}} R_{0} \simeq T_{1}^{2} \oplus T_{2}^{2}$.

LEMMA 4.10. Let $K_{3}$ be the minimal idempotent ideal of $R_{3}$ that is not contained in $\operatorname{Aug}\left(R_{3}\right)$. Then $\mathrm{QK}_{3}=e_{1} R_{0}+e_{5} R_{0}$. 
Proof. Put $e=1-e_{3}, g=(1,2)(3,4)$ and $h=(1,2,3,4,5)$. These elements of $G$ give idempotents $e^{\prime}=e\left(1-\frac{1}{2}(1+g)\right)$ and $f^{\prime}=e\left(1-\frac{1}{5}(1+h+\right.$ $\left.\left.+h^{2}+h^{3}+h^{4}\right)\right)$. Let $P^{\prime}=e^{\prime} R_{3}, P^{\prime \prime}=f^{\prime} R_{3}$ and $P=e R_{3}$. Let $M_{1}, M_{2}, M_{3}$ be the simple $R_{3}$-modules such that $M_{1}$ is a unique simple factor of $K_{3}, M_{2}$ is a unique simple factor of $e I_{3}$ and $M_{3}$ is a unique simple factor of $e_{3} R_{3}$. Again we want to find $k, l \in \mathbb{N}$ such that $P / P J\left(R_{3}\right) \simeq M_{1} \oplus M_{2}^{l}$ and $P^{\prime} / P^{\prime} J\left(R_{3}\right) \simeq M_{2}^{k}$.

Consider the module $M$ over $S_{3}$ given by the obvious action of $A_{5}$ on the vector space $\left\{\left(z_{1}, \ldots, z_{5}\right) \in Z_{3}^{5} \mid z_{1}+\cdots+z_{5}=0\right\}$ (that is, if $x \in A_{5}$, then $\left.\left(z_{1}, \ldots, z_{5}\right) x=\left(z_{x(1)}, \ldots, z_{x(5)}\right)\right)$. The module $M$ can be viewed as an absolutely simple representation of $A_{5}$ over $Z_{3}$ and its dimension is 4 . Now consider $M$ as an $R_{3}$-module via the canonical epimorphism $\pi: R_{3} \rightarrow S_{3}$. Then $M$ is a simple $R_{3}$-module annihilated by $e_{3}$, therefore $M \simeq M_{2}$. It follows that the multiplicity of $M_{2}$ in $R_{3} / J\left(R_{3}\right)$ is 4 , therefore $l=4$.

Since $P^{\prime}$ is a direct summand of $P, k \in\{1,2,3,4\}$. Using Fact 4.8, we get $\operatorname{dim}_{Z_{3}} P / P\left(3 R_{3}\right)=42, \operatorname{dim}_{Z_{3}} P^{\prime} / P^{\prime}\left(3 R_{3}\right)=18, \operatorname{dim}_{Z_{3}} P^{\prime \prime} / P^{\prime \prime}\left(3 R_{3}\right)=36$. Now the only simple factor of $P^{\prime}$ and $P^{\prime \prime}$ is $M_{2}$, so that $P^{\prime \prime} \simeq P^{\prime 2}$. Thus $P^{\prime 2}$ is a direct summand of $P$, and therefore $k \in\{1,2\}$. If $k=1$, then $P^{\prime 3}$ would be a direct summand of $P$ and this is not possible, because $42<3 \cdot 18$. Therefore $k=2$ and there exists $Q$ such that $P \simeq P^{\prime 2} \oplus Q$. The semisimple module $Q \otimes_{R_{3}} R_{0}$ has its Q-dimension equal to 6 and the multiplicity of $T_{1}$ in $Q \otimes_{R_{3}} R_{0}$ is 1 . The only possibility is $Q \otimes_{R_{3}} R_{0} \simeq T_{1} \oplus T_{5}$. Hence $\mathrm{Q} \operatorname{Tr}(Q)=e_{1} R_{0}+e_{5} R_{0}$.

LEMMA 4.11. Let $K_{2}$ be the minimal idempotent ideal of $R_{2}$ that is not contained in $\operatorname{Aug}\left(R_{2}\right)$. Then $\mathrm{Q} K_{3}=e_{1} R_{0}+e_{3} R_{0}+e_{5} R_{0}$.

Proof. Let $M_{1}, M_{2}, M_{3}$ be the simple $R_{2}$-modules such that $M_{1}$ is the simple factor of $K_{2}, M_{2}$ is the simple factor of $\left(1-e_{2}\right) I_{2}$ and $M_{3}$ is the simple factor of $e_{2} R_{2}$. Let $e=1-e_{2}, e^{\prime}=e\left(1-\frac{1}{3}\left(1+g+g^{2}\right)\right)$, where $g=(1,2,3)$. Put $P=e R_{2}, P^{\prime}=e^{\prime} R_{2}$, so that $P / P J\left(R_{2}\right) \simeq M_{1} \oplus M_{2}^{l}$ and $P^{\prime} / P^{\prime} J\left(R_{2}\right) \simeq M_{2}^{k}$.

Let $F$ be a field given by adjoining a primitive fifteenth root of one to $Z_{2}$. By [18, page 200], the ring $F \otimes S_{2} / J\left(S_{2}\right)$ has two 2-dimensional simple modules and they are annihilated by $e_{2}$ (because they appear as composition factors of a representation annihilated by $e_{2}$ ). Therefore $F \otimes M_{2}$ is a direct sum of these two representations. Thus the $Z_{2}$-dimension of $M_{2}$ is 4 , but the multiplicity of $M_{2}$ in $S_{2} / J\left(S_{2}\right)$ is 2 . It follows that $l=2$.

Using Fact 4.8, we get that the $Z_{(2)}$-rank of $P$ is 44 and $Z_{(2)}$-rank of $P^{\prime}$ is 32 . Therefore $P^{\prime 2}$ cannot be a direct summand of $P$ and $k=2$ follows. 
Then $P \simeq P^{\prime} \oplus Q$ for some $Q$ and $K_{2}=\operatorname{Tr}(Q)$. By Lemma 4.7, $\mathrm{Q} K_{2}=\operatorname{Tr}\left(Q \otimes_{R_{2}} R_{0}\right)$. Observe that $Q \otimes_{R_{2}} R_{0}$ has Q-dimension 12 and contains $T_{1}$ with multiplicity 1 . The only way of writing 11 as a sum of multiples of 6 and 5 is $11=6+5$. Therefore $Q \otimes_{R_{2}} R_{0} \simeq T_{1} \oplus T_{5} \oplus T_{3}$ and $\mathrm{Q} K_{2}=\left(e_{1}+e_{3}+e_{5}\right) R_{0}$.

Now we can finish the classification of the idempotent ideals in $Z\left[A_{5}\right]$.

Proposition 4.12. The idempotent ideals in $R=Z\left[A_{5}\right]$ are $0, \operatorname{Aug}(R), X$ and $R$, where $X \neq R$ and $\mathrm{Q} X=\mathrm{Q}\left[A_{5}\right]$.

Proof. The idempotent ideals contained in $\operatorname{Aug}(R)$ were classified in Lemma 4.5. Let $K$ be an idempotent ideal of $R$ not contained in $\operatorname{Aug}(R)$. Then for any $i \in\{2,3,5\}, K_{(i)}$ is an idempotent ideal of $R_{i}$ not contained in $\operatorname{Aug}\left(R_{i}\right)$. By Lemma 4.9 , we have $e_{2} \in K_{(0)}$, by Lemma 4.10 , we have $e_{5} \in K_{(0)}$ and by Lemma 4.11, we have $e_{3} \in K_{(0)}$. It follows that $K_{(0)}=\mathrm{Q}\left[A_{5}\right]$.

If $L$ is an idempotent ideal of $R_{5}$ such that $\mathrm{Q} L=\mathrm{Q}\left[A_{5}\right]$, then $L=R_{5}$ by Lemmas 4.5 and 4.9. Similarly, if $L$ is an idempotent ideal of $R_{3}$ such that $\mathrm{Q} L=\mathrm{Q}\left[A_{5}\right]$, then $L=R_{3}$ by Lemmas 4.5 and 4.10 . But if $L$ is an idempotent ideal of $R_{2}$ such that $\mathrm{Q} L=\mathrm{Q}\left[A_{5}\right]$, then either $L=R_{2}$ or $L=K_{2}+e_{2} R_{2}$ by Lemmas 4.5 and 4.11. Therefore there exists an idempotent ideal $X \subseteq R$ such that $X_{(2)}=K_{2}+e_{2} R_{2}, X_{(3)}=R_{3}$ and $X_{(5)}=R_{5}$.

Finally, we can classify the non-finitely generated projective modules over $\mathbb{Z}\left[A_{5}\right]$.

THEOREM 4.13. The countably but not finitely generated projective modules over $R=Z\left[A_{5}\right]$ are the following: Let $I=\operatorname{Aug}(R)$ and let $X$ be the other non-trivial idempotent ideal of $R$. Let $B_{I}$ be the unique I-big projective $R$-module with trace $I$, and let $B_{X}$ be the unique X-big projective module with trace $X$. Apart from these, there is an X-big projective module $P$ such that $P / P X$ is the unique indecomposable projective module over $R / X$. Then:

(i) Any countably generated projective module over $R$ that is neither free nor finitely generated has a unique decomposition as a sum $Q \oplus F$, where $Q \in\left\{B_{X}, B_{I}, P\right\}$ and $F$ is a finitely generated free module.

(ii) $B_{X} \oplus B_{I} \simeq R^{(\omega)}$ and $B_{I} \oplus P \simeq R^{(\omega)}$.

(iii) $P \oplus B_{X} \simeq P$ and $P \oplus P \simeq R \oplus B_{X}$. 
Proof. Let $M$ be a countably generated projective module over $R$. Since $R$ has (*), there exists a least ideal $K$ such that $M / M K$ is finitely generated. If $K=0, M$ is finitely generated. If $K=R$, then $M$ is $R$-big and hence free. If $K=I$, then $M / M I \simeq Z^{n}$ for some $n \in \mathbb{N}_{0}$, because $R / I \simeq Z$. Since $N=B_{I} \oplus R^{n}$ is a countably generated projective module such that $I$ is the smallest ideal of the set $\{L$ ideal of $R \mid N / N L$ is finitely generated $\}$ and $N / N I \simeq M / M I$, by Lemma 2.5 and Corollary 2.10 , we have $M \simeq N$. Clearly, for every $m, n \in \mathbb{N}$, one has $B_{I} \oplus R^{n} \simeq B_{I} \oplus R^{m}$ if and only if $m=n$.

The remaining case is $X=K$. Recall that $X_{(p)}=R_{p}$ for any prime different from 2. It follows that there exists $k \in \mathbb{N}$ such that $2^{k} \in X$. Now $R / X \simeq\left(R / 2^{k} R\right) /\left(X / 2^{k} R\right) \simeq\left(R_{2} / 2^{k} R_{2}\right) /\left(X_{(2)} / 2^{k} R_{2}\right)$. Let $S=Z_{2^{k}}\left[A_{5}\right]$, let $\pi: R_{2} \rightarrow S$ be the canonical epimorphism and let $X^{\prime}=\pi\left(X_{(2)}\right)$. From the proof of Lemma 4.11, we know that $S / J(S) \simeq M_{1} \oplus M_{2}^{2} \oplus M_{3}^{n}$ for some $n \in \mathbb{N}$ (in fact $n=4$, but we do not need this) and the $M_{1}, M_{3}$ are the simple factors of $X^{\prime}$. Now $S / J(S) /\left(X^{\prime}+J(S)\right) / J(S) \simeq\left(S / X^{\prime}\right) /\left(J\left(S / X^{\prime}\right)\right) \simeq \mathrm{M}_{2}\left(\operatorname{End}_{S}\left(M_{2}\right)\right)$. It follows that $R / X$ is a homogeneous semilocal ring with an indecomposable projective module $P^{\prime}$ satisfying $P^{\prime 2} \simeq R / X$. The module $P^{\prime}$ gives a unique countably generated projective module $P$ such that $P$ is $X$-big and $P / P X \simeq P^{\prime}$. Since $P^{\prime} \oplus P^{\prime} \simeq R / X$, we get $P \oplus P \simeq B_{X} \oplus R$. The relation $B_{X} \oplus P \simeq P$ holds because $P$ is $X$-big.

It remains to prove the relations in (ii). Since a direct sum of an $X$-big module and an $I$-big module is $R$-big, these relations follow immediately.

\section{One more application.}

Finally let us consider universal enveloping algebras. Let $\mathrm{g}$ be a Lie algebra over a field $\mathrm{k}$ and let $X$ be a basis of $\mathrm{g}$. A universal enveloping algebra of $\mathrm{g}$, denoted by $U(\mathrm{~g})$, is a factor of the free k-algebra over $X$ modulo the relations $x y-y x=[x, y](x, y \in X)$. If $\mathrm{g}$ is a nilpotent Lie algebra of finite dimension, then $U(\mathrm{~g})$ is a left and right noetherian ARdomain (see [11, Section 4.2] for the definition). It follows that all infinitely generated projective modules are free [12, Lemma 8.6]. The AR-property does not hold for solvable Lie algebras in general, but property (*) does. This enables us to prove that infinitely generated projective modules are free over $U(\mathrm{~g})$ if $\mathrm{g}$ is a solvable Lie algebra of finite dimension and $\mathrm{k}$ has characteristic zero. This concludes the proof of [12, Conjecture 8.5], stating that a finite dimensional Lie algebra over a field of characteristic zero is 
solvable if and only if any (left and right) projective module over $U(\mathrm{~g})$ is a direct sum of finitely generated modules.

We say that a ring $R$ satisfies strong (*) if every sequence of ideals $I_{1}, I_{2}, \ldots \subseteq R$ satisfying $I_{k+1} I_{k}=I_{k+1}, k \in \mathbb{N}$ has either $I_{k}=R$ for every $k \in \mathbb{N}$ or there exists $l \in \mathbb{N}$ such that $I_{l}=0$. Let us point out the following straightforward consequence of Bass' theorem [3, Theorem 3.1].

LEMma 5.1. Let $R$ be a left and right noetherian ring satisfying (*). Then the following are equivalent:

(i) $R$ satisfies strong (*).

(ii) The only idempotent ideals of $R$ are 0 and $R$.

(iii) Every projective module over $R$ is either finitely generated or free.

LEMma 5.2. Let $S$ be a noetherian domain and let $D: S \rightarrow S$ be a derivation on $S$. Let $R=S_{D}[x]$ be the corresponding skew polynomial ring. If $X$ and $Y$ are ideals of $R$ such that $X Y=X$ and $X$ is nonzero, then $Y$ contains a constant polynomial.

Proof. $\quad$ Let $K$ be the (left and right) quotient field of $S$ and $\bar{D}: K \rightarrow K$ the derivation extending $D$. Then $R$ can be considered as a subring of the (left and right) principal ideal domain $\bar{R}=K_{\bar{D}}[x]$. Let $\bar{X}$ be the ideal of $\bar{R}$ generated by $X$ and let $\bar{Y}$ be the ideal of $\bar{R}$ generated by $Y$. Using the division algorithm one can check that $\bar{X}=\left\{s^{-1} p \mid 0 \neq s \in S, p \in X\right\}$ and $\bar{Y}=\left\{p s^{-1} \mid 0 \neq s \in S, p \in Y\right\}$. Considering the degrees of the polynomials, $\bar{X} \neq 0$ implies $\bar{Y}=\bar{R}$. But then $Y$ must contain a polynomial of degree 0.

Proposition 5.3. Let $S$ be a noetherian prime algebra over $Q$ satisfying strong (*). Suppose that $D: S \rightarrow S$ is a derivation on $S$ and $R=S_{D}[x]$ is the corresponding skew polynomial ring. If every prime ideal of $S$ is completely prime, then $R$ satisfies strong (*).

Proof. Let $I_{1}, I_{2}, \ldots$ be a sequence of nonzero ideals in $R$ such that $I_{k+1} I_{k}=I_{k+1}$ for every $k \in \mathbb{N}$. We have to prove that $I_{k}=R$ for every $k \in \mathbb{N}$. For any ideal $I \subseteq R$, consider the smallest ideal $c(I)$ of $S$ such that $I \subseteq \sum_{i=0}^{\infty} c(I) x^{i}$. Observe that $c\left(I_{k+1}\right) c\left(I_{k}\right)=c\left(I_{k+1}\right)$ and $c\left(I_{k}\right) \neq 0$ for every $k \in \mathbb{N}$. Therefore the strong (*) in $S$ implies $c\left(I_{k}\right)=S$ for every $k \in \mathbb{N}$.

Now let $Q$ be a prime ideal of $S$ invariant under $D$. On $S / Q$ define $D_{Q}: S / Q \rightarrow S / Q$ by $D_{Q}(s+Q):=D(s)+Q, s \in S$. Consider the ring 
$R_{Q}=S / Q_{D_{Q}}[x]$ and the canonical projection $\pi_{Q}: R \rightarrow R_{Q}$. Observe that $\pi_{Q}$ is an epimorphism with kernel $Q^{\prime}=\sum_{i=0}^{\infty} Q x^{i}$.

We claim that for any prime ideal $Q \subseteq S$ invariant under $D$ and for any $k \in \mathbb{N}$ we have $\pi_{Q}\left(I_{k}\right)=R_{Q}$. Then we conclude applying the claim to $Q=0$.

Suppose the claim is not true that is the set $M=\{Q \mid Q$ is a prime ideal of $R$ invariant under $D$ such that $\pi_{Q}\left(I_{l}\right) \neq R_{Q}$ for some $\left.l \in \mathbb{N}\right\}$ is nonempty. Let $P$ be a maximal ideal of $M$. Let $\pi: S \rightarrow S / P$ be the canonical projection. Observe that $P$ cannot be a maximal two-sided ideal of $S$ : Since $c\left(I_{k}\right)=S, \pi_{P}\left(I_{k}\right) \neq 0$ for every $k \in \mathbb{N}$. Applying Lemma 5.2 to $\pi_{P}\left(I_{1}\right), \pi_{P}\left(I_{2}\right), \ldots$ we get $S / P \cap \pi_{P}\left(I_{k}\right) \neq 0$. Therefore if $S / P$ is a simple ring, then $1 \in \pi_{P}\left(I_{k}\right)$ for every $k \in \mathbb{N}$.

In general, Lemma 5.2 gives $L_{k}=\pi_{P}\left(I_{k}\right) \cap S / P \neq 0$. Put $L_{k}^{\prime}=\pi^{-1}\left(L_{k}\right)$ and notice that $L_{k}^{\prime}$ is an ideal of $S$ invariant under $D$. If $L_{k}^{\prime}=S$ for every $k \in \mathbb{N}$, then $\pi_{P}\left(I_{k}\right)=R_{P}$ for every $k \in \mathbb{N}$, a contradiction to the choice of $P$. Therefore suppose that $L_{l}^{\prime} \neq S$ for some $l \in \mathbb{N}$. Let $P_{1}, \ldots, P_{m}$ be the minimal primes of $L_{l}^{\prime}$. As $S$ is a Q-algebra, applying [6, Lemma 3.3.3], $P_{1}, \ldots, P_{m}$ are primes of $S$ invariant under $D$ properly containing $P$. In particular, $\pi_{P_{i}}\left(I_{l}\right)=R_{P_{i}}$ or $R=I_{l}+P_{i}^{\prime}$ for every $i=1, \ldots, m$. Then $R=\left(I_{l}+P_{1}^{\prime}\right) \cdots\left(I_{l}+P_{m}^{\prime}\right)$ $=I_{l}+P_{1}^{\prime} \cdots P_{m}^{\prime}$ also. Further, by [11, Theorem 2.3.7], there exists $n \in \mathbb{N}$ such that $\left(P_{1} \cdots P_{m}\right)^{n} \subseteq L_{l}^{\prime}$. Note $R=R^{n}=I_{l}+\left(P_{1}^{\prime} \cdots P_{m}^{\prime}\right)^{n}$, therefore $R_{P}=\pi_{P}(R) \subseteq \pi_{P}\left(I_{l}\right)+R_{P} L_{l} R_{P}=\pi_{P}\left(I_{l}\right)$. So $R_{P}=\pi_{P}\left(I_{l}\right)$, a contradiction again.

LEMMA 5.4. Let $\mathrm{k}$ be a field of characteristic zero and let $\mathrm{g}$ be a solvable Lie algebra of finite dimension over $\mathrm{k}$. Then $\mathrm{U}(\mathrm{g})$ satisfies strong (*).

Proof. First suppose that $\mathrm{k}$ is algebraically closed. Then $\mathrm{g}$ is completely solvable by [11, Theorem 14.5.3]. That is, there exists a basis $x_{1}, \ldots, x_{n}$ of $\mathrm{g}$ over $\mathrm{k}$ such that $\mathrm{g}_{m}=\mathrm{k} x_{1}+\cdots+\mathrm{k} x_{m}$ is an ideal of $\mathrm{g}$ for every $m=1, \ldots, n$. Then $U\left(\mathbf{g}_{m+1}\right)$ can be seen as a skew polynomial ring over $U\left(\mathrm{~g}_{m}\right)$ for $m=1, \ldots, n-1$.

Recall that each prime ideal of $U\left(\mathrm{~g}_{m}\right)$ is completely prime by [11, Theorem 14.2.11], therefore we can apply Proposition 5.3.

In general, let $\overline{\mathrm{k}}$ be an algebraic closure of $\mathrm{k}$. Let $I_{1}, I_{2}, \ldots$ be a sequence of nonzero ideals in $U(\mathrm{~g})$ such that $I_{k+1} I_{k}=I_{k+1}$ for every $k \in \mathbb{N}$. Consider $\bar{R}=U(\mathrm{~g}) \otimes \overline{\mathrm{k}} \simeq U(\mathrm{~g} \otimes \overline{\mathrm{k}})$ and the ideals $\overline{I_{k}}=I_{k} \otimes \overline{\mathrm{k}}$. It is easy to see that $\overline{I_{k+1}}=\overline{I_{k+1} I_{k}}$ for every $k \in \mathbb{N}$. By the preceding step, $\overline{I_{k}}=\bar{R}$ for every $k \in \mathbb{N}$. But this is possible only if $I_{k}=U(\mathrm{~g})$. 
Corollary 5.5. Let $\mathrm{g}$ be a finite dimensional solvable Lie algebra over a commutative field of characteristic zero. Then

(i) Every idempotent ideal of $U(\mathrm{~g})$ is trivial.

(ii) The universal enveloping algebra of $\mathrm{g}$ satisfies (*).

(iii) Every projective $U(\mathrm{~g})$-module that is not finitely generated is free.

Acknowledgments. The author is extremely grateful to Gena Puninski for his comments and suggestions and to the referee for improving the presentation of this paper.

\section{REFERENCES}

[1] T. Akasaki, Idempotent ideals of integral group rings, J. Algebra, 23 (1972), pp. 343-346.

[2] F. W. ANdERSON - K. R. Fuller, Rings and categories of modules, Springer Verlag, 1974.

[3] H. BAss, Big projective modules are free, Illinois J. Math. (1963), pp. 24-31.

[4] R. CAMPS - W. DiCKS, On semilocal rings, Israel J. Math., 81 (1993), pp. 203-211.

[5] C. W. Curtis - I. Reiner, Methods of representation theory with applications to finite groups and orders, Vol. 1, Wiley-Interscience (1981).

[6] J. Dixmier, Enveloping algebras, Akademie - Verlag (Berlin, 1977).

[7] A. HatToRI, Rank element of a projective module, Nagoya Math. J., 25 (1965), pp. 113-120.

[8] H. Kraft - L. W. Small - N. R. Wallach, Properties and examples of FCRalgebras, Manuscripta math., 104 (2001), pp. 443-450.

[9] V. D. MAZurov - E. I. KHUKHro, The Kourovka notebook. Unsolved problems in group theory, 15th augm. ed, Novosibirsk Institut Matematiki (2002).

[10] T. Y. LAM, A first course in noncommutative rings, Springer, New York, 2001.

[11] J. C. MCConnell - J. C. RoBson, Noncommutative noetherian rings, AMS, Providence, R. I., 2001.

[12] G. PUnInski, When a projective module is a direct sum of finitely generated modules, preprint, 2004.

[13] P. PříHodA, Projective modules are determined by their radical factors, J. Pure Appl. Algebra, 210 (2007), pp. 827-835.

[14] K. W. RogGenkamP, Integral group rings of solvable finite groups have no idempotent ideals, Arch. Math., 25 (1974), pp. 125-128.

[15] L. W. Small - J. C. RoBson, Idempotent ideals in P.I. rings, Journal London Math. Soc. (2), 14 (1976), pp. 120-122.

[16] R. G. Swan, Induced representations and projective modules, Ann. of Math., 71 (1960), pp. 552-578.

[17] R. G. Swan, The Grothendieck ring of a finite group, Topology, 2 (1963), pp. $85-110$.

[18] J. M. Whitehead, Projective modules and their trace ideals, Comm. Algebra, 8 (19) (1980), pp. 1873-1901.

Manoscritto pervenuto in redazione l'1 aprile 2009. 
